\title{
AVALIAÇÃO CAROTÍDEA EM DOENTES SUBMETIDOS A REVASCULARIZAÇÃO MIOCÁRDICA
}

\author{
CAROTID EVALUATION OF PATIENTS UNDERGOING CORONARY \\ ARTERY BYPASS GRAFT
}

\author{
Luiz Roberto Felizzola ${ }^{1}$ \\ Ana Terezinha Guillaumon, TCBC-SP ${ }^{2}$
}

\begin{abstract}
RESUMO: Objetivo: Como a doença carotídea é a principal causa isolada de acidente vascular cerebral de origem tromboembólica, acredita-se ser imprescindível o seu diagnóstico precoce. O presente estudo tem por objetivo realizá-lo, mantendo os conceitos de praticidade e viabilidade econômica, adequando-se às condições socioeconômicas desfavoráveis locais. Método: No período de 18 meses foram avaliados 50 doentes, sendo 35 homens e 15 mulheres, com média de idade de 67,2 anos. Todos apresentavam indicação de revascularização miocárdica, considerados coronariopatas graves. Avaliou-se a incidência e o grau de estenose carotídea com base no mapeamento dúplex. Da mesma forma, foi avaliada a relação com possíveis fatores de risco: diabetes melito, hipertensão arterial sistêmica, tabagismo, sintomatologia neurológica focal prévia, doença arterial troncular dos membros inferiores e presença de sopro carotídeo. Resultados: A incidência de estenose carotídea hemodinamicamente significativa ( $>50 \%$ ) foi de $48 \%$, e crítica (>70\%) de $32 \%$. Mostraram-se fatores de risco estatisticamente significativos, antecedentes de diabetes melito, sintomatologia neurológica focal prévia, presença de sopro carotídeo e presença de alterações arteriais tronculares dos membros inferiores. Conclusão: Concluiu-se que no grupo estudado houve aumento significativo de risco para estenose carotídea hemodinamicamente significativa e crítica, em relação à população geral. Desta forma, justifica-se o rastreamento ultrasonográfico para doença carotídea em doentes coronariopatas, principalmente quando os fatores de risco significativos se fizerem presentes.
\end{abstract}

Descritores: Ultra-sonografia Doppler; Isquemia cerebral; Artéria carótida; Isquemia miocárdica.

\section{INTRODUÇÃO}

A importância da doença carotídea como freqüente fator causal para acidente vascular cerebral já está claramente demonstrada, estando da mesma forma satisfatoriamente delineados seu tratamento clínico, indicações e técnica cirúrgica. Todavia, a preocupação com o seu diagnóstico e tratamento parece ainda incipiente em nosso meio, tendo em vista a baixa freqüência com que a en- darterectomia carotídea é realizada, se comparada à literatura estrangeira.

Dentre os vários fatores que poderiam levar a esta situação, seriam principais a dificuldade de acesso aos meios diagnósticos efetivos, e a provável descrença na prevalência atribuída à estenose carotídea.

O estudo ultra-sonográfico com avaliação colorida de fluxo pelo efeito Doppler (mapeamento dúplex) veio solucionar a dificuldade de acesso ao diagnóstico. Por não

1. Mestre em Cirurgia pela UNICAMP. Responsável pelo Serviço de duplex ultra-som da PUC-Campinas. Responsável pelo Serviço de duplex ultra-som do InCOR-Sorocaba

2. Professora-Assistente da Disciplina de Moléstias Vasculares Periféricas do Depto. de Cirurgia - UNICAMP, Coordenadora do Núcleo de Medicina e Cirurgia Experimental e do Laboratório de Microprocedimentos e Pesquisa Vascular FCM - UNICAMP

Recebido em 9/3/2000

Aceito para publicação em 30/01/2001

Trabalho desenvolvido como dissertação para obtenção do título de Mestre em Cirurgia, pela UNICAMP, defendido e aprovado em julho de 1998. 
apresentar riscos, ser acessível economicamente, apresentar elevadas sensibilidade e especificidade além de disponibilidade freqüente, este método vem rapidamente substituindo a arteriografia no rastreamento da doença carotídea e até mesmo como exame único na avaliação pré-operatória para endarterectomia carotídea.

Uma vez que, como é sabido, a doença aterosclerótica apresenta caráter generalizado, acometendo os vários territórios arteriais em grau variável, torna-se razoável inferir que doentes com aterosclerose manifesta em um determinado território teriam maior probabilidade de apresentá-la de forma significante nos demais.

Partindo desta prerrogativa, no sentido de identificar grupo de alto risco para doença carotídea, justificando e preconizando seu rastreamento, desenvolveu-se o presente estudo onde foram avaliados doentes com coronariopatia grave quanto à presença de estenose carotídea.

Entendeu-se por coronariopatia grave aquela com indicação de revascularização miocárdica. Tal interpretação teve o objetivo de conferir às conclusões do presente estudo aplicação clara e prática, evitando-se a freqüente utilização de extratificações complexas e de domínio quase exclusivo do cardiologista, fato determinante de dificuldade na aplicação de outros estudos.

\section{MÉTODO}

O presente estudo selecionou pacientes com coronariopatia isquêmica grave, entendendo-se por grave aquela com indicação de revascularização miocárdica, a serem avaliados sob o aspecto de presença de doença carotídea estenosante. Comparou-se então os achados deste estudo com a incidência da doença na população geral já determinada pela bibliografia consultada.

Desta forma, por via telefônica ou telegrama, convocaram-se doentes que aguardavam por cirurgia de revascularização miocárdica a comparecer ao ambulatório de Cirurgia Vascular do Hospital das Clínicas de Campinas UNICAMP, onde os mesmos foram esclarecidos do propósito de sua convocação, contemplando-se os critérios da Portaria 196/96 do Ministério da Saúde.

Compareceram e aceitaram a proposta deste estudo 50 doentes, 35 do sexo masculino e 15 do sexo feminino, com idade média de 67,2 anos (41 a 89 anos), no período de agosto de 1996 a janeiro de 1998. Procedeu-se, então, à avaliação vascular através de anamnese dirigida, registro de eventuais fatores de risco para doença carotídea como antecedentes pessoais, hábitos e antecedentes familiares, e exame físico vascular completo, tendo sempre o cuidado de ser realizado pelo mesmo observador, para manutenção do padrão.

$\mathrm{Na}$ anamnese dirigida deu-se especial ênfase aos sintomas de doença cérebro-vascular extracraniana, sendo estes: amaurose fugaz, acidente isquêmico transitório (AIT), e acidente vascular cerebral isquêmico (AVCI). Não foram valorizados sintomas como vertigens, tonturas e síncopes, devido à sua inespecificidade para doença carotídea, sendo mais freqüentes na doença vascular vertebrobasilar, labirintopatias, e doenças que cursam com baixo débito cardíaco ${ }^{1,2}$.
Com relação aos antecedentes e hábitos pessoais, inquiriu-se sobre diabetes melito, dislipidemias, insuficiência renal, infarto do miocárdio, acidente vascular cerebral, endarterectomia carotídea prévia, tabagismo, aneurismas arteriais, cirurgias vasculares arteriais prévias e amputações por doença isquemiante. Inquiriu-se ainda sobre antecedentes familiares de infarto agudo do miocárdio e acidente vascular cerebral.

No que tange aos antecedentes, 14 (28\%) apresentavam diabetes melito (DM), $25(50 \%)$ hipertensão arterial sistêmica (HAS), 27 (54\%) tabagismo, 22 (44\%) dislipidemia, $10(20 \%)$ história familiar de acidente vascular cerebral (AVC) e $16(32 \%)$ de infarto agudo do miocárdio (IAM).

Os doentes foram submetidos à ultra-sonografia com avaliação colorida de fluxo pelo efeito Doppler (mapeamento dúplex) das artérias carótidas comuns, internas e externas no segmento cervical, sempre realizado pelo mesmo examinador. Utilizaram-se como parâmetros de avaliação para eventuais estenoses a comparação entre a imagem à secção transversal pelo modo B (diminuição percentual do diâmetro do lúmen carotídeo em relação ao real diâmetro da carótida interna pós-bulbar), e alterações das velocidades sistólica e diastólica, à secção longitudinal.

Com relação à avaliação da estenose através das alterações de velocidades, foram utilizados os parâmetros que se seguem: critérios de velocidade de Dawson \& Strandness Jr. e critérios de imagem ao modo B de SallesCunha \& Andross 2,3 .

Sempre com a preocupação da aplicabilidade do estudo, embasado nos critérios citados, dividiu-se a casuística segundo planejamento terapêutico definido na literatura consultada. Desta forma, os doentes foram separados em: portadores de estenoses não hemodinamicamente significativas ( $0 \%$ a $49 \%$ ), portadores de estenoses hemodinamicamente significativas (superior a 50\%), portadores de estenoses críticas (70\% a 99\%) e oclusão. Para inclusão do doente nos grupos citados utilizou-se o maior grau de estenose entre a carótida interna direita e esquerda. Quando foi encontrada oclusão total de uma destas considerouse o grau de estenose contralateral para incluir o doente num dos grupos citados. Somente foram incluídos no grupo oclusão, aqueles com oclusão total bilateral.

Optou-se por definir estenose crítica como aquela superior a $70 \%$ por ser o critério usado pelo NASCET, estudo multicêntrico mais valorizado em nosso meio ${ }^{4}$.

Sete doentes foram submetidos a exames complementares posteriores, para confirmação dos achados ultra-sonográficos, por opção do profissional que lhes assistia previamente. Destes, seis foram submetidos à carotidoangiografia e um à angiorressonância nuclear magnética, havendo confirmação dos achados ultra-sonográficos na totalidade.

\section{RESULTADOS}

Dos 50 doentes avaliados, 26 (52\%) foram incluídos no grupo estenoses não hemodinamicamente significati- 
vas ( $0 \%$ a 49\%), 24 (48\%) foram incluídos no grupo estenoses hemodinamicamente significativas (superior a 50\%), sendo $8(16 \%)$ estenoses moderadas $(50 \%$ a $70 \%)$ e 16 (32\%) incluídos no grupo estenoses superiores a $70 \%$ (estenoses críticas), nenhum doente apresentava oclusão bilateral, conforme pode ser observado no Gráfico I. mente significativa em portadores de antecedentes neurológicos focais.

Ao exame físico encontrou-se pulsos carotídeos na totalidade dos casos, sopro carotídeo em 13 doentes (26\%) e doença arterial troncular dos membros inferiores em 21 doentes $(42 \%)$.

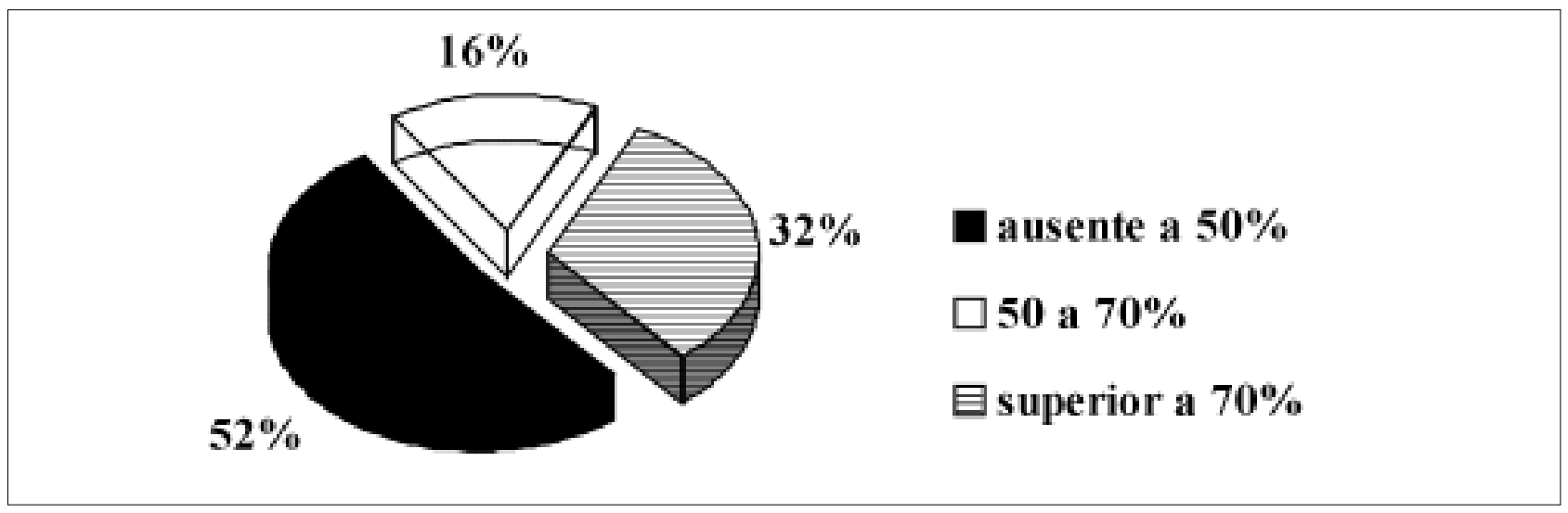

Gráfico 1 - Distribuição da doença carotídea segundo o grau de estenose em coronariopatas graves.

Com relação ao parâmetro sexo, houve diferenças em relação aos antecedentes pessoais e familiares, sendo mais evidentes o tabagismo, presente em $13,3 \%$ das mulheres e $71,4 \%$ dos homens, e história familiar de infarto agudo do miocárdio presente em $20 \%$ das mulheres e $54,3 \%$ dos homens. Embora tenha sido observada diferença na incidência de doença carotídea crítica entre os sexos feminino $(13,3 \%)$ e masculino (42\%), tal diferença não se mostrou estatisticamente significativa $(\mathrm{p}=0,11)$.

Dos antecedentes pessoais e familiares, apenas o diabetes melito demonstrou ser significante fator de risco para doença carotídea em coronariopatas graves $(\mathrm{p}<0,008)$. A medida odds ratio demonstrou risco 21 vezes maior de estenose carotídea hemodinamicamente significativa em coronariopatas graves com diabetes melito do que os coronariopatas não diabéticos.

A sintomatologia neurológica focal esteve presente em 17 (34\%) dos doentes, sendo 4 (8\%) devido a AVC, 8 $(16 \%)$ a AIT e $6(12 \%)$ a amaurose fugaz. Doentes sintomáticos apresentaram incidência de estenose hemodinamicamente significativa da ordem de $76,5 \%$, e crítica de $47,1 \%$. Dentre os assintomáticos $33,3 \%$, apresentaram estenose hemodinamicamente significativa e $24,2 \%$ crítica.

Desta forma, os antecedentes neurológicos focais apresentaram sensibilidade de 54,2\%, especificidade de $84,6 \%$, índice prognóstico positivo de $76,5 \%$ e negativo de $66,7 \%$, para o diagnóstico de estenose hemodinamicamente significativa. Com relação à estenose crítica, apresentaram sensibilidade de $50 \%$, especificidade de $73,5 \%$, índice prognóstico positivo de 73,5\% e negativo de $75,6 \%$. A análise estatística comprovou a significância deste fator de risco $(\mathrm{p}<0,05)$, sendo que a medida odds ratio demonstrou risco 8 vezes maior de estenose hemodinamica-
O sopro carotídeo esteve relacionado à estenose hemodinamicamente significativa em $11(84,6 \%)$ dos doentes que apresentavam sopro carotídeo. Em contrapartida, apenas $11(45,8 \%)$ dos 24 doentes com estenose superior a $50 \%$ apresentavam sopro carotídeo.

A presença de sopro carotídeo apresentou sensibilidade de $45,8 \%$, especificidade de $92,3 \%$, índice prognóstico positivo de $57,1 \%$ e negativo de $86,2 \%$ para estenose hemodinamicamente significativa. Já para estenose crítica apresentou sensibilidade de 56,3\% especificidade de $88,2 \%$, índice prognóstico positivo de $69,2 \%$ e negativo de $81,1 \%$, correspondendo assim a fator de risco estatisticamente significativo ( $\mathrm{p}<0,006)$. A análise da medida odds ratio demonstrou que doentes com sopro carotídeo apresentam risco 13 vezes maior para estenose hemodinamicamente significativa e 15 vezes maior para estenose crítica.

A doença arterial troncular dos membros inferiores esteve associada à estenose hemodinamicamente significativa em $81 \%$ dos doentes, apresentando sensibilidade de $70,8 \%$, especificidade de $84,6 \%$, valor preditivo positivo de $81 \%$ e valor preditivo negativo de $75,9 \%$. Quando considerada estenose crítica apresentou sensibilidade de $75 \%$, especificidade de $73,5 \%$, valor preditivo positivo de $57,1 \%$ e valor preditivo negativo de $86,2 \%$.

As alterações arteriais tronculares dos membros inferiores demonstraram-se fator de risco estatisticamente significativo para estenose carotídea moderada $(\mathrm{p}<0,02)$ e crítica $(\mathrm{p}<0,003)$ em coronariopatas graves. A análise da medida odds ratio indicou risco nove vezes maior de estenose hemodinamicamente significativa e 17 vezes maior de estenose carotídea crítica. 


\section{DISCUSSÃO}

Dados epidemiológicos norte-americanos vieram demonstrar ser a doença carotídea a principal causa isolada de acidente vascular cerebral, o qual atinge a cifra de 500.000 casos novos ao ano, respondendo pela terceira causa de morte naquele país ${ }^{5}$. Embora não possuindo dados estatísticos nacionais, os autores acreditam serem transponíveis tais dados estatísticos para a nossa realidade.

Grandes estudos multicêntricos vieram estabelecer a real contribuição do tratamento da doença carotídea, seja de forma clínica, com o controle dos fatores de risco para aterosclerose e uso de antiagregantes plaquetários, seja através do tratamento cirúrgico, por meio da endarterectomia carotídea. Os estudos "NASCET", "VETERANS", "ACAS", "ECST" preconizaram o tratamento clínico para doentes com estenoses incipientes (inferiores a $30 \%$ ), e tratamento cirúrgico, por meio da endarterectomia carotídea, nas estenoses críticas (superiores a $70 \%$ ). Estenoses entre 30 e $60 \%$ ainda não apresentam normatização definida, devendo receber abordagem individualizada, caso a caso. Todavia, o tratamento preconizado é preferencialmente clínico ${ }^{4,6-8}$.

Apesar de preconizado tratamento preferencialmente clínico para estenoses inferiores a 70\%, Johnson et al referem ser necessário seguimento ultra-sonográfico semestral, devido à progressão para estenose superior a $80 \%$ ocorrer em até $16 \%$ dos doentes, em seguimento de seis anos. Referem ainda incidência de $25 \%$ de AVC nos doentes que apresentam progressão neste período; em contrapartida apenas $1 \%$ dos doentes sem progressão apresentam $\mathrm{AVC}^{9}$.

Uma vez compreendida e valorizada a doença carotídea, restou a esta geração estabelecer de forma clara e prática quais os grupos de pacientes com risco elevado para doença carotídea, desta forma justificando seu rastreamento. Assim sendo, concentrar-se-ia o rastreamento sobre a população de maior risco, por ser economicamente inviável o rastreamento na população geral ${ }^{10}$.

Sabendo-se que a doença aterosclerótica possui padrão generalizado, acometendo em grau variável todos os territórios arteriais, torna-se natural acreditar que doentes com doença aterosclerótica manifesta em outros territórios apresentariam maior risco de doença carotídea, constituindo assim, população de risco ${ }^{11,12}$.

No sentido de comprovar esta hipótese, vários estudos foram conduzidos correlacionando a presença de doença aterosclerótica nos diversos territórios: membros inferiores, aórtico, coronariano e carotídeo, comprovando tal expectativa $^{11-15}$.

Embora a relação entre coronariopatia e doença carotídea, objetivo deste estudo, seja freqüentemente relatada na literatura consultada, não há uma concordância global de dados. Tal discordância se deve às diferentes estratificações da gravidade da doença coronariana, critérios de inclusão variáveis para doença carotídea, e princi- palmente a utilização de métodos diagnósticos com sensibilidades díspares.

Exemplificando esta dificuldade pode-se citar os estudos de Babu et al, que, utilizando a oculopletismografia, referem incidência de $0,26 \%$ de doença carotídea grave em coronáriopatas, e de Mand'ak et al., que, utilizando o mapeamento duplex, relatam incidência de 59,4\% de doença carotídea de gravidade variável ${ }^{12,16}$.

Todavia, quando foram selecionados estudos com método diagnóstico coincidente, houve relativa concordância de dados, estimando a incidência de estenose carotídea hemodinamicamente significativa $(>50 \%)$ entre $12,7 \%$ e $17,02 \%$, e estenose crítica (>80\%) entre $6 \%$ e $16 \%$; mesmo assim, nem sempre a extratificação da doença coronariana foi clara ${ }^{17,18}$.

O objetivo do presente estudo foi quantificar a presença e qualificar o grau de doença carotídea de maneira clara e prática em doentes submetidos à revascularização miocárdica, considerando-os coronariopatas graves, fixando-se assim a variável grau de coronariopatia. Adotandose critérios de extratificação para doença carotídea que pudessem levar à aplicação direta na terapêutica, dividiuse a amostra em quatro subgrupos, segundo o grau de estenose: ausente ou inferior a 50\%, superior a 50\% (hemodinamicamente significativo). superior a $70 \%$ (crítica) e oclusão.

Tal subdivisão se deve à conduta já relativamente estabelecida e citada anteriormente, onde o subgrupo com estenose ausente ou inferior a $50 \%$ seria submetido a tratamento clínico, estenose superior a $70 \%$ a tratamento cirúrgico, e o subgrupo intermediário seria seguido por avaliação ultra-sonográfica semestral para identificação de eventual progressão da doença $a^{4,6-9}$.

O motivo pelo qual somente doentes com oclusão carotídea bilateral seriam incluídos no subgrupo oclusão se deve ao fato de que após a oclusão o doente não corre mais riscos de acidentes vasculares cerebrais de causa carotídea ipsilateral. Desta forma, apenas a eventual estenose contralateral implicaria em tais riscos, determinando assim a conduta a ser seguida.

Embora haja na literatura relatos de redução na incidência de acidente vascular cerebral no per-operatório de revascularização miocárdica, em doentes submetidos a tratamento prévio da doença carotídea, não foi objetivo deste estudo avaliar tal fenômeno ${ }^{19,20}$.

Da mesma forma não foi objetivo deste estudo avaliar a sobrevida de pacientes submetidos à revascularização miocárdica que recebem tratamento para doença carotídea prévio ou concomitante à revascularização miocárdica, embora haja relato de aumento de sobrevida e melhora da qualidade de vida neste grupo ${ }^{18}$.

O método diagnóstico escolhido para este estudo foi o mapeamento duplex devido à sua elevada sensibilidade (90\% a 98\%) e especificidade (próxima de 95\%), baixo custo, ausência de riscos, e possível melhor correlação com os achados ao estudo anatomopatológico ${ }^{21-23}$.

A opção pela não utilização da carotidoangiografia como método diagnóstico segue a tendência atual de 
considerar proibitivo o risco de acidente vascular cerebral de $1 \%$ a $4 \%$, se comparado ao risco peroperatório de $1 \%$ a $5 \%$ da endarterectomia carotídea ${ }^{5,22,24-27}$.

Embora não fazendo parte do protocolo deste estudo, sete $(14 \%)$ dos 50 doentes avaliados foram submetidos posteriormente a outros exames para ratificação do resultado do mapeamento duplex, por opção do profissional que lhes assistia previamente. Utilizou-se a carotidoangiografia em seis e a angiorressonância nuclear magnética em um doente, havendo concordância dos achados na totalidade dos casos.

Deve-se ressaltar que os dados encontrados foram comparados a estudos estrangeiros por não ter sido encontrada referência nacional que fornecesse os dados desejados. Embora sempre seja recomendável a comparação a grupo controle, em vez de estudos publicados, não foi realizado grupo controle pela inviabilidade técnica de obter amostra não viciada, sendo necessário para tanto, a avaliação de um número extremamente elevado de voluntários, inviável para as condições técnicas desta instituição.

A incidência de estenose carotídea crítica foi de $32 \%$ no grupo estudado, $42 \%$ em homens e $13,3 \%$ em mulheres, mostrou-se significativamente elevada em relação à incidência na população geral acima de quarenta anos, a qual é de $2,4 \%$ em homens e $1,1 \%$ em mulheres (28). Embora o estudo referido faça referência a importância da faixa etária, o presente estudo não demonstrou tais diferenças de modo estatisticamente significativo.

Da mesma forma, tal incidência foi elevada mesmo se comparada a outros estudos em coronariopatas, onde se encontrou $6 \%$ a $16 \%$ de estenose crítica e $12,7 \%$ a $17 \%$ de estenose hemodinamicamente significativa . Este fato pode expressar melhora no método diagnóstico, ou ainda estar relacionado à maior gravidade dos doentes com acesso a esta instituição ${ }^{17,18}$.

Não houve significância estatística como fatores de risco para doença carotídea em coronariopatas graves, quanto ao sexo, idade, antecedentes de tabagismo, dislipidemia, hipertensão arterial, antecedentes familiares de infarto agudo do miocárdio ou acidente vascular cerebral.

Embora a diferença de incidência em homens e mulheres seja aparentemente grande, esta não se apresentou estatisticamente significativa no presente estudo, provavelmente devido a casuística reduzida. O mesmo pode ser verdade em relação a fatores de risco clássicos como o tabagismo, dislipidemia e idade.

Mostraram-se estatisticamente significativos os fatores: antecedentes pessoais de diabetes melito, sintomatologia neurológica prévia, presença de sopro carotídeo e presença de doença arterial troncular dos membros inferiores. Tais dados vão ao encontro dos referidos na literatura ${ }^{1,12-15,28-30}$.
No que tange ao exame físico, a presença do sopro carotídeo foi indicativa de estenose hemodinamicamente significativa em $84,6 \%$ dos doentes e crítica em $69,2 \%$, dados mais expressivos que os obtidos na população geral, onde a incidência de estenose significativa em portadores de sopro carotídeo é da ordem de 37,5 a $50 \%{ }^{12}$. Esta melhor correlação provavelmente deve-se ao fato de estarem sendo avaliados doentes realmente com sopro carotídeo, diferenciando-se este do sopro cervical, utilizado como parâmetro em vários estudos. Para tanto o examinador tomou o cuidado de se certificar que não se tratava de sopro cardíaco irradiado, frequientemente observado.

Todavia, apenas $45,8 \%$ dos doentes portadores de estenose carotídea apresentavam-se com sopro; incidência próxima da literatura, onde cerca de $33 \%$ das estenoses significativas apresentam sopro ${ }^{18}$. Assim sendo, permanece não aconselhável a utilização deste parâmetro para a exclusão da doença carotídea devido sua baixa sensibilidade.

Ao se avaliar a presença de sintomatologia neurológica focal, obteve-se associação à estenose significativa em $76,5 \%$ dos doentes e crítica em $47,1 \%$ dos mesmos. Porém, $33,3 \%$ dos doentes assintomáticos apresentaram estenose significativa e $24,2 \%$ crítica, da mesma forma não permitindo considerar sua ausência parâmetro confiável de normalidade quanto ao território carotídeo.

O fato das alterações arteriais tronculares dos membros inferiores apresentarem sensibilidade 75\%, especificidade $73,53 \%$, valor preditivo positivo $57,14 \%$ e negativo $86,2 \%$ para estenoses críticas, leva a considerá-las o fator de risco mais importante para doença carotídea crítica em coronariopatas graves, ratificando os achados de Salasidis et al ${ }^{14}$. Todavia, os dados obtidos também não permitem descartar doença carotídea significante, pela ausência de doença arterial troncular dos membros inferiores.

Os doentes com coronariopatia grave estudados apresentaram significativo aumento na incidência de doença estenosante carotídea moderada e grave se comparados à população geral.

Os achados que isoladamente apresentaram-se estatisticamente significativos indicadores de doença estenosante carotídea moderada ou grave em coronariopatas graves foram: antecedente pessoal de diabetes melito, sintomatologia neurológica focal prévia, presença de sopro carotídeo e presença de doença arterial dos membros inferiores.

A presença de doença arterial dos membros inferiores foi o principal fator de risco isolado para doença carotídea em coronariopatas graves.

Desta forma, acredita-se ser justificado o rastreamento da doença carotídea em portadores de coronariopatia grave, especialmente quando associada à doença arterial troncular dos membros inferiores. 


\begin{abstract}
Purpose: Carotid disease is known as the main individual cause of stroke. It is necessary to develop means to achive an early diagnosis using economically viable methods. Method: During 18 months, 50 patients with coronary disease and were indication for coronary artery bypass evaluated (35 male, 15 female; mean age of 67.2 years). The presence and degree of carotid artery stenosis was determined with duplex ultrasonography. The association of carotid artery disease and diabetes mellitus, arterial systemic hypertension, smoking, previous focal neurologic symptoms, lower extremity arterial disease and cervical bruit, was also determined. Results: It was found that $48 \%$ of the patients presented hemodynamically significant carotid stenosis (>50\%) and 32\% of showed critic carotid stenosis (>70\%). Diabetes mellitus, previous focal neurological symptoms, cervical bruit and lower extremity arterial disease were considered significant risk factors. These results clearly showed that there was a significant increase in the risk of presenting hemodynamically significant or critical carotid stenosis in these group of patients compared to the rest of the population. Conclusion: In conclusion, the use of ultrasonographic screening for carotid disease, in patients suffering from coronary artery disease, is very justified, mainly when significant risk factors are present.
\end{abstract}

Key Words: Doppler ultrasonography; Cerebral isquemia; Carotid artery; Miocardial isquemia.

\section{REFÊRÊNCIAS}

1. Hill SL, Holtzman GL, Berry R, Arnold JF - The appropriate use of the duplex scan in carotid arterial disease. Am. Surg. 1997; v.63, n.8, p.720-5.

2. Salles-Cunha SX, Andros G - Extracranial cerebrovasculature in the neck.. In: Salles-Cunha, S.X., Andros, G. Atlas of duplex ultrasonography: Essential images of the vascular system. California. Appleton Davies, 1988; p.3-50.

3. Dawson DL, Strandness Jr. DE - Duplex scanning. In: Strandness Jr. DE \& van Breda A - Vascular diseases: surgical and interventional therapy. New York: Churchill Livingstone, 1994; p.157-85.

4. North american symptomatic carotid endarterectomy trial collaborators - Beneficial effect of carotid endarterectomy in symptomatic patients with high-grade carotid stenosis. N. Engl. J. Med. 1991; v.325, p.44553.

5. Nicholas GG, Osborne MA, Jaffe JW, Reed JF - Carotid artery stenosis: preoperative noninvasive evaluation in a community hospital. J. Vasc. Surg. 1995; v.22, p.9-16.

6. Hobson RW, Weiss DG, Fields WS - Efficacy of carotid endarterectomy for asymptomatic carotid stenosis. N. Engl. J. Med. 1993; v. 328, p.221-7.

7. Executive committe for the asyntomatic carotid atherosclerosis study. Endarterectomy for asymptomatic carotid artery stenosis. JAMA 1995; v.273, p.1421-8.

8. European carotid surgery trialists' collaborative group. MCR European carotid surgery trial: interim results for symptomatic patients with severe (70-99\%) or with mild (0-29\%) carotid stenosis. Lancet 1991; n. 337, p.1235-43.

9. Johnson BF, Verlato F, Bergelin RO, Primozich JF, Strandness DE - Clinical outcome in patients with mild and moderate carotid artery stenosis. J. Vasc. Surg. 1995; v. 21, p.120-6.

10. Lee TT, Solomon NA, Heidenreich PA, Oehlert J, Garber AM - Cost-effectiveness of screening for carotid stenosis in asymptomatic persons. Ann. Intern. Med. 1997; v. 126, n.5, p.337-46.
11. Del Bosque VP - Valoración de las lesiones coronarias y carotídeas asociadas a los aneurismas de aorta infrarenal. In: Estevan-Solano, J.M. - Tratado de aneurismas. Barcelona: J. Uriach, 1997; p. 305-18.

12. Mand'ak J, Lonsky V, Dominic J, Harrerova L, Brtko M Concomitant coronary and asymptomatic carotid artery disease in patients prior to myocardial revascularization. Acta Medica Hradec. Kralove. 1996; v. 39, n.1, p.31-4.

13. D'Agostino RS, Svensson LG, Neumann DJ et al. - Screening carotid ultrasonography and risk factors for stroke in coronary artery surgery patients. Ann. Thorac. Surg 1996; v. 62, n. 6, p. 1714-23.

14. Salasidis GC, Latter DA, Steinmetz OK, Blair JF, Graham AM - Carotid artery duplex scanning in preoperative assesment for coronary artery revascularization: the association between peripheral vascular disease, carotid artery stenosis, and stroke. J. Vasc. Surgery. 1995; v. 21, n.1, p.154-62.

15. Virgilio C, Toosie K, Arnell T et al. - Asymptomatic carotid artery stenosis screening in patients with lower extremity atherosclerosis: a prospective study. Ann. Vasc. Surg. 1997; v. 11, n.4, p.374-7.

16. Babu SC, Shah PM, Singh BM, Semel LS, Clauss RH, Reed GE - Coexisting carotid stenosis in patients undergoing cardiac surgery: indications for simultaneous operations. Am. J. Surg. 1985; v.150, n.2, p.207-11.

17. Lusiani L, Visona A, Castellani V et al. - Prevalence of atherosclerotic lesions of the carotid bifurcation found with echo-doppler (duplex) technic in patient candidates for aortocoronary bypass. G. Ital. Cardiol. 1986; v.16, n.2, p.103-13.

18. Loop FD - Changing management of carotid stenosis in coronary artery surgery patients. Ann. Thorac. Surg. 1988; v. 45, p. 591-2.

19. Ricotta JJ, Faggioli GL, Castilone A, Hassett JM - Risk factors for stroke after cardiac surgery: Buffalo CardiacCerebral study group. J. Vasc. Surg. 1995; v.21, n.2, p.359-63.

20. Schwartz LB, Bridgman AH, Kieffer RW et al. - Asymptomatic carotid artery stenosis and stroke in patients undergoing cardiopulmonar bypass. J. Vasc. Surg. 1995; v.21, p.146-53. 
21. Kirsch JD, Wagner LR, James EM et al. - Carotid artery occlusion: Positive predictive value of duplex sonography compared with arteriography. J. Vasc. Surg. 1994; v.19, p.642-9.

22. Kuntz KM, Skillman JJ, Whittemore AD, Kent C - Carotid endarterectomy in asymptomatic patients: is contrast angiography necessary? A morbidity analysis. J. Vasc. Surg. 1995 ; v. 22, p. 706-16.

23. Pan XM, Saloner D, Reilly LM et al. - Assessment of carotid artery stenosis by ultrasonography, conventional angiography: correlation with ex vivo measurement of plaque stenosis. J. Vasc. Surg. 1995; v. 21, p. 82-9.

24. Garrard CL, Manord JD, Ballinger BA et al. - Cost savings associated with the nonroutine use of carotid angiography. Am. J. Surg. 1997; v. 174, n. 6, p. 650-3.

25. Khaw KT - Does carotid duplex imaging render angiography redundant before carotid endarterectomy? $\mathrm{Br}$. J. Radiol. 1997; v. 70, p. 235-8.
26. Moreira RCR, Goes Jr. C.A, Stanishesk IC et al. - Endarterectomia de carótida semarteriografia: Experiência inicial. Cir. Vasc. Angiol. 1996; v. 12, p. 161-5.

27. Yates GN, Bergamini TM, George JR et al. - Carotid endarterectomy results from a state vascular society. Kentucky Vascular Surgery Society Study Group. Am. J. Surg. 1997; v. 173, n. 4, p. 342-4.

28. Willeit J, Kiechl S - Prevalence and risk factors of asymptomatic extracranial carotid aretry atherosclerosis: a population-based study. Arterioscler. throb. 1993; v. 13(5), p. 661-8.

29. Mackaay AJ, Beks PJ, Dur AH et al. - The distribution of peripheral vascular disease in a Dutch caucasian population: comparison of type II diabetic and non-diabetic subjects. Eur. J. Vasc. Endovasc. Surg. 1995; v.9, n.2, p.170-5.

30. Valentine RJ, Hagino RT, Boyd PI, Kakish HB, Clagett GP - Utility of carotid duplex in young adults with lower extremity atherosclerosis: how aggressive should we be in screening young patients? Cardiovasc. Surg. 1997; v. 5, n. 4, p. 408-13. 